\title{
A biased random-key genetic algorithm for the minimization of open stacks problem
}

\author{
José Fernando Gonçalves ${ }^{\mathrm{a}}$, Mauricio G. C. Resende ${ }^{\mathrm{b}}$ and Miguel Dias Costa ${ }^{\mathrm{c}}$ \\ ${ }^{a}$ LIAAD, INESC TEC, Faculdade de Economia do Porto, Universidade do Porto, Rua Dr. Roberto Frias, s/n, 4200-464 \\ Porto, Portugal \\ ${ }^{\mathrm{b}}$ AT\&T Labs Research, 180 Park Avenue, Room C241, Florham Park, NJ 07932, USA \\ ${ }^{\mathrm{c}}$ Faculdade de Economia do Porto, Universidade do Porto, Rua Dr. Roberto Frias, s/n, 4200-464 Porto, Portugal \\ E-mail: jfgoncal@fep.up.pt [Gonçalves]; mgcr@research.att.com [Resende]; migueldiascosta@gmail.com [Costa]
}

Received 27 July 2013; received in revised form 27 May 2014; accepted 27 May 2014

\begin{abstract}
This paper describes a biased random-key genetic algorithm $(B R K G A)$ for the minimization of the open stacks problem $(M O S P)$. The $M O S P$ arises in a production system scenario, and consists of determining a sequence of cutting patterns that minimize the maximum number of open stacks during the cutting process. The proposed approach combines a $B R K G A$ and a local search procedure for generating the sequence of cutting patterns. A novel fitness function for evaluating the quality of the solutions is also developed. Computational tests are presented using available instances taken from the literature. The high quality of the solutions obtained validate the proposed approach.
\end{abstract}

Keywords: minimization of open stacks problem; cutting pattern; biased random-key genetic algorithm; random keys

\section{Introduction}

Cutting stock problems consist in cutting smaller pieces (items) from larger pieces (objects) and arise in many industrial production scenarios, such as the furniture, paper, steel, and wood hardboard industries. In the solution of cutting stock problems, we seek to minimize waste or maximize profit through the selection of a set of good cutting patterns. However, in certain cases, it is also important to determine the sequence in which the set of cutting patterns should be processed so as to minimize the maximum stack of partially cut orders. A cutting stock solution defines a set of cutting patterns and the number of times the patterns have to be cut to satisfy the demand for the items. When the patterns are cut, the items cut are piled up in stacks, one stack for each item type. The first time an item type is cut, a stack is considered "open." It remains open until the last piece of the corresponding item type is cut. A stack is "closed" when the last piece of an item type is cut. Because of space availability or equipment limitations, which may force some stacks to be removed to free 
Table 1

Data for illustrative example MOSP

\begin{tabular}{ll}
\hline Items & Patterns containing items \\
\hline A & 1,3 \\
B & $1,2,4$ \\
C & 1,2 \\
D & 3 \\
E & 2,4 \\
\hline
\end{tabular}

(a) - $M$

\begin{tabular}{|c|c|c|c|c|c|}
\hline & \multicolumn{4}{|c|}{ Patterns } \\
\hline & & 1 & 2 & 3 & 4 \\
\hline \multirow{5}{*}{ Items } & $\mathbf{A}$ & 1 & 0 & 1 & 0 \\
\hline & B & 1 & 1 & 0 & 1 \\
\hline & c & 1 & 1 & 0 & 0 \\
\hline & D & 0 & 0 & 1 & 0 \\
\hline & E & 0 & 1 & 0 & 1 \\
\hline
\end{tabular}

(b) - $M^{1}$

Fig. 1. $M$ and $M^{1}$ corresponding to permutation $s=(1,2,3,4)$ for the example of Table 1.

up space for the new stacks, it is desirable to maintain a small number of open stacks during the cutting process. Closed stacks can be moved to another location or delivered to clients. If removed, open stacks must be later returned so that work can be completed. This is inefficient since it takes time and uses scarce resources. To avoid the inefficiencies caused by the removal and later return of open stacks, it is important to determine the optimal cutting order of the patterns such that the maximum number of open stacks during the cutting process is minimized. This problem is known as the minimization of open stacks problem $(M O S P)$. To illustrate the $M O S P$, we use an example problem with five item types and four patterns. This problem is detailed in Table 1.

Yanasse and Senne (2010) define MOSP as follows: Let $M$ be a Boolean matrix, where each row corresponds to an item type and each column corresponds to a cutting pattern. Each entry of $M_{i, k}$ (with $i=1, \ldots, n$ and $k=1, \ldots, m$ ) equals 1 if and only if at least one item of type $i$ is contained in pattern $k$. Let $M_{s}^{1}$ be the resulting matrix corresponding to the permutation $s$ of the columns of $M$ such that in any row of $M_{s}^{1}$, each 0 entry between two 1 entries are replaced by a 1 . Figure 1 depicts matrices $M$ and $M^{1}$ corresponding to the permutation $s=(1,2,3,4)$ for the example presented in Table 1.

The objective of MOSP is to find a permutation $s^{*}$ of the columns, such that the maximum number of 1 entries in any column of matrix $M_{s^{*}}^{1}$ is minimized. Figure 2 depicts one optimal solution for the example. It corresponds to the permutation $s=(3,1,2,4)$ and has an $M O S P$ value of three.

Though the interest of the operations research community in the MOSP increased after the realization of the 2005 Constraint Modeling Challenge in the Fifth Workshop on Modeling and 


\section{Patterns}

\begin{tabular}{|c|c|c|c|c|c|}
\hline & & 3 & 1 & 2 & 4 \\
\hline \multirow{5}{*}{ Items } & A & 1 & 1 & 0 & 0 \\
\hline & B & 0 & 1 & 1 & 1 \\
\hline & C & 0 & 1 & 1 & 0 \\
\hline & D & 1 & 0 & 0 & 0 \\
\hline & $\mathbf{E}$ & 0 & 0 & 1 & 1 \\
\hline
\end{tabular}

Op. Stacks $2 \quad 3 \quad 3 \quad 2$

Fig. 2. $M^{1}$ corresponding to the optimal permutation $s=(3,1,2,4)$ for the example in Table 1.

Solving Problems with Constraints, which focused on the MOSP, the number of publications on this problem is still not extensive.

The special case of MOSP, where there are at most two different item types per pattern, was considered by Lins (1989). While trying to solve a problem faced by the Australian glass industry, Yuen $(1991,1995)$ developed six simple heuristics for the MOSP. The third heuristic (called Heuristic 3) was considered the most efficient in computational tests. Yuen and Richardson (1995) proposed the simple lower bound for the MOSP of the maximum number of different item types in the patterns and an exact method that enumerates permutations of pattern sequences. The new lower bound and upper bounds provided by the heuristics of Yuen $(1991,1995)$ were used to reduce the search space for the exact method. Yanasse (1996) proposes polynomial time algorithms for MOSP instances with special topologies. Yanasse (1997b), Limeira (1998), and Yanasse and Limeira (2004) propose branch-and-bound algorithms to solve the MOSP. Yanasse (1997a) defines the MOSP as a graph problem and shows that any MOSP instance corresponding to the same MOSP graph are equivalent. Faggioli and Bentivoglio (1998) develop a mathematical formulation for the MOSP and solution method involving three phases. The first phase finds a good solution with a greedy heuristic similar to some of the heuristics of Yuen (1995). The second phase improves the solution obtained in the first phase using a tabu search. The third phase uses an implicit enumeration scheme of the permutations of patterns. Yanasse et al. (1999) and Becceneri (1999) propose arc contraction heuristics. A new lower bound for the optimal value of the MOSP is presented in Yanasse et al. (1999). Becceneri (1999) proposed the least-cost node heuristic for the MOSP, which was later modified in Becceneri et al. (2004).

Metaheuristic-based heuristics have also been used to solve the MOSP. A simulated annealing heuristic is proposed in Linhares et al. (1999), and simulated annealing and tabu search are used in Fink and Voß (1999). A constructive genetic algorithm (GA) is proposed in Oliveira and Lorena (2002). Yanasse et al. (2007) proposed exact and heuristic methods using properties of the solution of MOSP to establish partial orders in which the nodes in the graph should be closed. An adaptive GA for large-sized open stack problems is presented in De Giovanni et al. $(2010,2013)$.

Dynamic programming solutions to MOSP were developed by Banda and Stuckey (2007) and Chu and Stuckey (2009), where the search was simplified through the use of the properties presented in Becceneri et al. (2004) and Yuen and Richardson (1995). 
Problems equivalent to the MOSP can arise in completely different contexts such as VLSI design (the gate matrix layout problem, one-dimensional logic, and PLA folding) and graph theory (interval thickness, node search game, edge search game, graph path-width, narrowness, split bandwidth, edge separation, and vertex separation; see Linhares and Yanasse, 2002; Möhring, 1990). Yanasse and Senne (2010) present a review of some properties and their use in preprocessing operations for the MOSP.

The MOSP is known to be NP-hard (Linhares and Yanasse, 2002). Therefore, when large instances are considered, heuristics are often the methods of choice. In this paper, we present a novel biased random-key GA (BRKGA) for the MOSP. The proposed algorithm hybridizes a local search procedure with a GA based on random keys. The BRKGA is used to evolve the order in which the patterns are inserted in a partial solution. To evaluate the quality of the solutions, a novel fitness function is also developed.

The remainder of the paper is organized as follows. In Section 2, we introduce the new approach, describing in detail the BRKGA, local search procedure, and novel fitness function. Finally, in Section 3, we report computational experiments, and in Section 4 we make concluding remarks.

\section{BRKGA}

In this section, we present an overview of the proposed solution process. This is followed by a discussion of the BRKGA, including detailed descriptions of the solution encoding and decoding, evolutionary process, fitness function, and parallel implementation.

\subsection{Overview}

The new approach is based on a constructive heuristic algorithm that inserts patterns, one at a time, in a partial pattern sequence for the problem. Once all the patterns are inserted, a solution is obtained. The new approach proposed in this paper combines a BRKGA, local search procedure, and novel fitness function. The role of the GA is to evolve the encoded solutions, or "chromosomes", which represent the "pattern insertion sequence" $(P I S)$. For each chromosome, the following phases are applied to decode the chromosome:

(1) Decoding of the PIS: This first phase decodes the chromosome into the PIS, that is, the sequence in which the patterns are inserted into the partial pattern sequence.

(2) Construction of a solution: The second phase makes use of the PIS defined in phase 1 and a local search procedure to construct a pattern sequence solution.

(3) Chromosome adjustment: The third phase adjusts the genes of the chromosome to reflect the changes made in phase 2.

(4) Fitness evaluation: The final phase computes the fitness of the solution (or measure of quality of the solution). For this phase, we developed a novel measure of fitness that significantly improves the quality of the solutions.

Figure 3 illustrates the sequence of decoding steps applied to each chromosome generated by the BRKGA. The remainder of this section describes in detail the GA, decoding procedure, local search, and adjustment procedure. 




Fig. 3. Architecture of the algorithm.

\section{2. $B R K G A$}

"Random-key GAs" (RKGAs) or GAs with random keys were introduced in Bean (1994) for solving sequencing or optimization problems whose solutions can be represented as permutations. In an RKGA, chromosomes are represented as vectors of randomly generated real numbers in the interval $[0,1]$. A deterministic algorithm, the "decoder", takes as input a chromosome and associates with it a solution of the combinatorial optimization problem for which an objective value or fitness can be computed.

RKGAs are particularly attractive for sequencing problems and/or when the chromosomes have several parts (see, e.g. Gonçalves and Almeida, 2002; Gonçalves and Resende, 2004, 2012, 2013; Gonçalves et al., 2005, 2009; Gonçalves and Sousa, 2011; Morán-Mirabal et al., 2014). Unlike the traditional GAs that use special repair procedures to handle permutations or sequences, RKGAs move all the feasibility issues to the objective evaluation procedure and guarantee that all offspring formed by crossover are feasible solutions. When the chromosomes have several parts, traditional GAs need to use different genetic operators for each part. However, since RKGAs use the "parametrized uniform crossover" of Spears and Dejong (1991) (instead of the traditional one-point or two-point crossover), they do not need to have different genetic operators for each part.

An $R K G A$ evolves a "population" of random-key vectors over a number of "generations" (iterations). The initial population is made up of $p$ vectors of $r$ random keys. Each component of the solution vector, or random key, is generated independently at random in the real interval $[0,1]$. After the fitness of each individual is computed by the decoder in generation $g$, the population is divided into two groups of individuals: a small group of $p_{e}$ "elite" individuals, that is, those with the best 
fitness values, and the remaining set of $p-p_{e}$ "nonelite" individuals. To evolve a population $g$, a new generation of individuals is produced. All elite individuals of the population of generation $g$ are copied without modification to the population of generation $g+1$. $R K G A$ s implement mutation by introducing "mutants" into the population. A mutant is a vector of random keys generated in the same way in which an element of the initial population is generated. At each generation, a small number $p_{m}$ of mutants is introduced into the population. With $p_{e}+p_{m}$ individuals accounted for in population $g+1, p-p_{e}-p_{m}$ additional individuals need to be generated to complete the $p$ individuals that make up population $g+1$. This is done by producing $p-p_{e}-p_{m}$ offspring solutions through the process of "mating" or "crossover."

A "BRKGA" (Gonçalves and Resende, 2011) differs from an $R K G A$ in the way parents are selected for mating. While in the $R K G A$ of Bean (1994) both parents are selected at random from the entire current population, in $B R K G A$ s each element is generated combining a parent selected at random from the elite partition in the current population and one is selected at random from the rest of the population. Repetition in the selection of a mate is allowed, and therefore an individual can produce more than one offspring in the same generation. As in $R K G A \mathrm{~s}$, parameterized uniform crossover is used to implement mating in $B R K G A$ s. Let $\rho_{e}$ be the probability that the vector component of an elite parent is inherited by the offspring. For $i=1, \ldots, r$, the $i$ th component $c(i)$ of the offspring vector $c$ takes on the value of the $i$ th component $e(i)$ of the elite parent $e$ with probability $\rho_{e}$ and the value of the $i$ th component $\bar{e}(i)$ of the nonelite parent $\bar{e}$ with probability $1-\rho_{e}$.

When the next population is complete, the corresponding fitness values are computed for all the newly created random-key vectors and the population is divided into elite and nonelite individuals to start a new generation.

A $B R K G A$ searches the solution space of the combinatorial optimization problem indirectly by searching the $r$-dimensional continuous hypercube, using the decoder to map solutions in the hypercube to solutions in the solution space of the combinatorial optimization problem, where the fitness is evaluated.

To specify a BRKGA, we simply need to specify how solutions are encoded and decoded, and how their corresponding fitness values are computed. Next we specify our algorithm by first showing how the solutions of an MOSP are encoded and then decoded, and how their fitness evaluation is computed.

\section{Chromosome representation and decoding}

A chromosome encodes a solution to the problem as a vector of random keys. In a direct representation, a chromosome represents a solution of the original problem and is called "genotype," while in an indirect representation it does not, and special procedures are needed to obtain from it a solution called a "phenotype." In the present context, the solutions will be represented indirectly by parameters that are later used by a decoding procedure to obtain a solution. To obtain the solution (phenotype), we use the decoding procedures described in Section "Solution builder."

In this paper, a solution to the MOSP is represented indirectly by the following chromosome structure:

$$
\text { chromosome }=\left(\text { gene }_{1}, \ldots, \text { gene }_{m}\right),
$$

where $m$ is the number of patterns. The decoding (mapping) of the $m$ genes of each chromosome into a PIS, which will be used by the solution builder (see Section "Solution builder"), is accomplished 


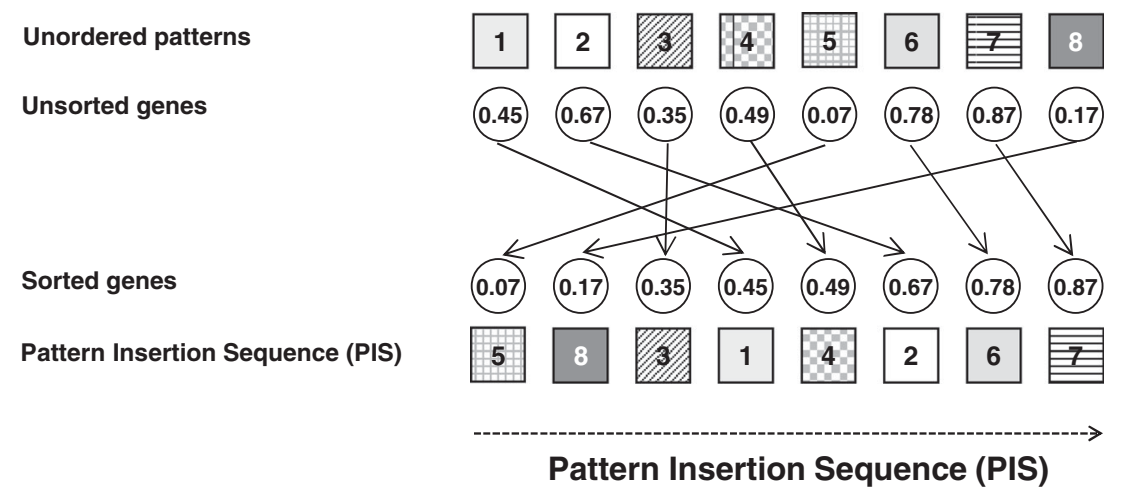

Fig. 4. Decoding of the pattern insertion sequence (PIS).

by sorting the patterns in an ascending order of the corresponding gene values. Figure 4 shows an example of the decoding process for the PIS. In this example, there are eight patterns. The sorted genes correspond to the $P I S=(5,8,3,1,4,2,6,7)$.

\section{Solution builder}

The solution builder follows a sequential process that inserts patterns into a partial solution, one pattern at each stage. The order in which the patterns are inserted into the partial solution is defined by the PIS evolved by the $B R K G A$. Each stage comprises the following two main steps:

(1) Selection of pattern to be inserted.

(2) Selection of the insertion position in the partial solution of the pattern selected in step 1).

The pattern selected for insertion at each stage $j$ is given by $P I S_{j}$. The position in the partial solution, where pattern $P I S_{j}$ will be inserted, is defined by a local search procedure. Let $m_{j}$ be the number of patterns already in the partial solution at stage $j$. Then the local search procedure considers the insertion of pattern $P I S_{j}$ before all existing patterns in positions $l=1, \ldots, j-1$ and after the pattern in position $j-1$. The insertion position $l_{j}^{*}$, corresponding to the smallest value of MOSP, is selected as the insertion position. Pattern $P I S_{j}$ is inserted at position $l_{j}^{*}$ and the process is repeated until all the patterns are inserted.

\section{Chromosome adjustment}

Solutions produced by the local search procedure usually disagree with the genes initially supplied to the decoder to obtain the PIS. Changes in the order of the patterns made by the local search phase of the decoder need to be taken into account in the chromosome. The heuristic adjusts the chromosome to reflect these changes. To make the chromosome supplied by the GA agree with the solution produced by local search, the heuristic adjusts the order of the genes according to the position of each pattern in the final solution. This chromosome adjustment not only improves the quality of the solutions but also reduces the number of generations needed to obtain the best values. 
(a) $-M^{1}$ for $s=(1,2,3,4)$

Patterns

\begin{tabular}{|c|c|c|c|c|c|}
\hline & & 1 & 2 & 3 & 4 \\
\hline & A & 1 & 1 & 1 & 0 \\
\hline & B & 1 & 1 & 1 & 1 \\
\hline Items & c & 1 & 1 & 0 & 0 \\
\hline & D & 0 & 0 & 1 & 0 \\
\hline & E & 0 & 1 & 1 & 1 \\
\hline
\end{tabular}

Op. Stacks $\quad 3 \quad 4 \quad 4 \quad 2$

MMOSP $=$ MOSP $+(3+4+4+2) /(4 \times 4)$

$=4.8125$ (b) $-M^{1}$ for $s=(2,1,4,3)$

Fig. 5. Example of the calculation of MMOSP.

\section{Fitness function}

The evolutionary process requires a measure of solution fitness, or quality measure. A natural fitness function for the MOSP is the "maximum number of open stacks" MOSP in a solution. However, since different solutions can have the same MOSP value, this measure does not differentiate well the potential for improvement of solutions having the same MOSP value.

To better differentiate the potential for improvement, we propose a new measure of fitness that we call "modified maximum number of open stacks," or simply MMOSP. The MMOSP combines $M O S P$ with a measure of the potential for improvement of a solution that has values in the interval $[0,1]$. The rationale for this new measure is that if we have two solutions that have the same MOSP value, then the one having the smallest average number of open stacks will have more potential for improvement.

Let $\operatorname{MOSP}_{K}$ be the number of open stacks when pattern $k$ is being cut. Let

$$
\frac{1}{m} \sum_{k=1}^{m} \operatorname{MOSP}_{k}
$$

be the average number of open stacks. Then, the value of the MMOSP is given by

$$
M M O S P=\operatorname{MOSP}+\frac{\sum_{k=1}^{m} \operatorname{MOSP}_{k}}{m \times M O S P} .
$$

The computational results in Section 3 show that this novel measure of fitness significantly improves the quality of the solutions. Figure $5 \mathrm{a}$ and b exemplifies the calculation of MMOSP for two solutions 
Table 2

Other approaches used for comparison

\begin{tabular}{lll}
\hline Approach & Type of method & Source of approach \\
\hline GHP & Greedy heuristic procedure & Faggioli and Bentivoglio (1998) \\
TS & Tabu search & Faggioli and Bentivoglio (1998) \\
GLS & Generalized local search & Faggioli and Bentivoglio (1998) \\
YUEN-3 and YUEN-5 & Heuristics & Yuen (1995) \\
DP1 & Dynamic programming & Banda and Stuckey (2007) \\
DP2 & Dynamic programming & Chu and Stuckey (2009) \\
CGA & Constructive genetic algorithm & Oliveira and Lorena (2002) \\
PMA & Parallel memetic algorithm & Mendes and Linhares (2004) \\
ECS & Evolutionary clustering search & Oliveira and Lorena (2006) \\
GRACS & Greedy randomized adaptive clustering search & Oliveira and Lorena (2006) \\
AS & Low-order polynomial time heuristic & Ashikaga and Soma (2009) \\
AGA & Adaptive genetic algorithm & De Giovanni et al. (2010, 2013) \\
\hline
\end{tabular}

having an MOSP value equal to 4. The first solution (Fig. 5a) has an MMOSP equal to 4.8125 and the second solution (Fig. 5b) has an MMOSP value of 4.75.

\section{Experimental results}

In this section, we report the results obtained on a set of experiments conducted to evaluate the performance of the BRKGA for MOSP proposed in this paper.

\subsection{Benchmark algorithms}

We compare $B R K G A$ with the literature approaches listed in Table 2.

\subsection{Test problem instances}

In the computational tests, we used three sets of instances described in Table 3.

\subsection{GA configuration}

The configuration of GAs is oftentimes more an art form than a science. In our past experience with GAs based on the same evolutionary strategy (see Gonçalves et al., 2005, 2009, 2011; Gonçalves and Resende, 2012, 2014), we obtained good results with values of TOP, BOT, and "crossover probability" (CProb) in the intervals shown in Table 4. 
Table 3

Benchmark instances used in the computational tests

\begin{tabular}{|c|c|c|c|}
\hline Set & Class & Description & Source \\
\hline & Harvey & $\begin{array}{l}2130 \text { random instances by Harvey. Three } \\
\text { benchmarks are proposed (denoted } \\
\text { "wbo," "wbop," and "wbp"), each } \\
\text { containing } 10 \text { classes }\end{array}$ & $\begin{array}{l}\text { Smith and Gent } \\
\text { (2005) }\end{array}$ \\
\hline & Simonis & $\begin{array}{l}3630 \text { random instances by Simonis, grouped } \\
\text { in } 10 \text { classes }\end{array}$ & $\begin{array}{l}\text { Smith and Gent } \\
\text { (2005) }\end{array}$ \\
\hline & Shaw & $\begin{array}{l}\text { One class of } 25 \text { random instances by Shaw, } \\
\text { with } 20 \text { patterns and item types }\end{array}$ & $\begin{array}{l}\text { Smith and Gent } \\
\text { (2005) }\end{array}$ \\
\hline \multirow[t]{4}{*}{$\begin{array}{l}\text { Literature } \\
\text { instances }\end{array}$} & $\begin{array}{l}\text { Miller and } \\
\text { Wilson }\end{array}$ & $\begin{array}{l}21 \text { individual instances, one provided by } \\
\text { Miller and } 20 \text { by Wilson (denoted GP } \\
1-8, \text { NWRS } 1-8 \text {, SP } 1-4)\end{array}$ & $\begin{array}{l}\text { Smith and Gent } \\
\text { (2005) }\end{array}$ \\
\hline & $\begin{array}{l}\text { Faggioli and } \\
\text { Bentivoglio }\end{array}$ & $\begin{array}{l}300 \text { random instances with } \\
n=10,20,30,40,50 \text { and } \\
m=10,15,20,25,30,40 . \text { Each of the } \\
n \times m \text { combinations was replicated } \\
10 \text { times }\end{array}$ & $\begin{array}{l}\text { Faggioli and } \\
\quad \text { Bentivoglio (1998) }\end{array}$ \\
\hline & VLSI & $\begin{array}{l}11 \text { individual instances from the VLSI } \\
\text { industry }\end{array}$ & Hu and Chen (1990) \\
\hline & SCOOP & $\begin{array}{l}24 \text { real instances from two woodcutting } \\
\text { companies (denoted "A" and "B") }\end{array}$ & $\begin{array}{l}\text { Available from } \\
\text { http://www.scoop- } \\
\text { project.net }\end{array}$ \\
\hline $\begin{array}{l}\text { Harder } \\
\quad \text { instances }\end{array}$ & Harder 150 & $\begin{array}{l}150 \text { harder instances generated using the } \\
\text { procedure detailed in (Chu and Stuckey, } \\
2009 \text { ) }\end{array}$ & $\begin{array}{l}\text { De Giovanni et al. } \\
\text { (2013) }\end{array}$ \\
\hline $\begin{array}{l}\text { Becceneri } \\
\text { instances }\end{array}$ & Becceneri & $\begin{array}{l}\text { Set of instances that covers the most } \\
\text { common practical industrial scenario } \\
\text { applications }\end{array}$ & Becceneri (1999) \\
\hline
\end{tabular}

Table 4

Range of parameters in past implementations

\begin{tabular}{lr}
\hline Parameter & Interval \\
\hline$T O P$ & $0.10-0.25$ \\
$B O T$ & $0.15-0.30$ \\
Crossover probability (CProb) & $0.70-0.80$ \\
\hline
\end{tabular}

Table 5

Configuration parameters for the $B R K G A$ algorithm

\begin{tabular}{ll}
\hline Parameter & Value \\
\hline$p$ & $10 \times m$ \\
$p_{e}$ & $\min (0.25 \times p, 50)$ \\
$p_{m}$ & $0.20 \times p$ \\
$\rho_{e}$ & 0.70 \\
Fitness & $M M O S P=$ modified MOSP (to minimize) \\
Stopping riterion & 100 generations \\
\hline
\end{tabular}

(C) 2014 The Authors.

International Transactions in Operational Research (c) 2014 International Federation of Operational Research Societies 
Table 6

Computational results: Harvey, Simonis, and Shaw benchmarks (aggregate results)

\begin{tabular}{|c|c|c|c|c|c|c|c|c|c|}
\hline & \multirow{2}{*}{$\frac{I}{n}$} & \multirow{2}{*}{$\frac{P}{m}$} & \multirow[b]{2}{*}{ Elements } & \multicolumn{2}{|c|}{ BRKGA } & \multicolumn{2}{|l|}{ AGA } & \multicolumn{2}{|l|}{ DP1 } \\
\hline & & & & MOSP & $T$ (seconds) & MOSP & $T$ (seconds) & MOSP & $T$ (seconds) \\
\hline \multirow[t]{10}{*}{ Simonis } & 10 & 10 & 550 & 8.0 & 0.00 & 8.0 & 0.00 & 8.0 & 0.00 \\
\hline & 10 & 20 & 550 & 8.9 & 0.00 & 8.9 & 0.01 & 8.9 & 0.00 \\
\hline & 15 & 15 & 550 & 12.9 & 0.00 & 12.9 & 0.02 & 12.9 & 0.00 \\
\hline & 15 & 30 & 550 & 14.0 & 0.00 & 14.0 & 0.10 & 14.0 & 0.00 \\
\hline & 20 & 10 & 220 & 15.9 & 0.00 & 15.9 & 0.03 & 15.9 & 0.00 \\
\hline & 20 & 20 & 550 & 18.0 & 0.00 & 18.0 & 0.11 & 18.0 & 0.01 \\
\hline & 30 & 10 & 220 & 24.0 & 0.00 & 24.0 & 0.05 & 24.0 & 0.00 \\
\hline & 30 & 15 & 110 & 26.0 & 0.00 & 26.0 & 0.11 & 26.0 & 0.01 \\
\hline & 30 & 30 & 220 & 28.3 & 1.30 & 28.3 & 0.61 & 28.3 & 0.72 \\
\hline & 40 & 20 & 110 & 36.4 & 0.43 & 36.4 & 0.33 & 36.4 & 0.10 \\
\hline Shaw & 20 & 20 & 25 & 13.7 & 0.00 & 13.7 & 0.34 & 13.7 & 0.01 \\
\hline \multirow[t]{10}{*}{ wbo } & 10 & 10 & 40 & 5.9 & 0.00 & 5.9 & 0.00 & 5.9 & 0.00 \\
\hline & 10 & 20 & 40 & 7.4 & 0.00 & 7.4 & 0.02 & 7.4 & 0.00 \\
\hline & 10 & 30 & 40 & 8.2 & 0.00 & 8.2 & 0.08 & 8.2 & 0.00 \\
\hline & 15 & 15 & 60 & 9.4 & 0.00 & 9.4 & 0.06 & 9.4 & 0.00 \\
\hline & 15 & 30 & 60 & 11.6 & 0.00 & 11.6 & 0.39 & 11.6 & 0.03 \\
\hline & 20 & 10 & 70 & 12.9 & 0.00 & 12.9 & 0.04 & 12.9 & 0.00 \\
\hline & 20 & 20 & 90 & 13.7 & 0.00 & 13.7 & 0.22 & 13.7 & 0.01 \\
\hline & 30 & 10 & 100 & 20.1 & 0.00 & 20.1 & 0.08 & 20.1 & 0.00 \\
\hline & 30 & 15 & 120 & 21.0 & 0.00 & 21 & 0.18 & 21.0 & 0.01 \\
\hline & 30 & 30 & 140 & 22.6 & 1.08 & 22.6 & 1.11 & 22.6 & 1.11 \\
\hline \multirow{10}{*}{ wbop } & 10 & 10 & 40 & 6.8 & 0.00 & 6.8 & 0.00 & 6.8 & 0.00 \\
\hline & 10 & 20 & 40 & 8.1 & 0.00 & 8.1 & 0.04 & 8.1 & 0.00 \\
\hline & 10 & 30 & 40 & 8.6 & 0.00 & 8.6 & 0.06 & 8.6 & 0.00 \\
\hline & 15 & 15 & 60 & 10.4 & 0.00 & 10.4 & 0.05 & 10.4 & 0.00 \\
\hline & 15 & 30 & 60 & 12.2 & 0.00 & 12.2 & 0.34 & 12.2 & 0.02 \\
\hline & 20 & 10 & 40 & 14.3 & 0.00 & 14.3 & 0.02 & 14.3 & 0.00 \\
\hline & 20 & 20 & 90 & 14.9 & 0.00 & 14.9 & 0.15 & 14.9 & 0.01 \\
\hline & 30 & 10 & 40 & 22.5 & 0.00 & 22.5 & 0.05 & 22.5 & 0.00 \\
\hline & 30 & 15 & 60 & 22.4 & 0.00 & 22.4 & 0.13 & 22.4 & 0.01 \\
\hline & 30 & 30 & 140 & 23.8 & 0.00 & 23.9 & 0.95 & 23.8 & 0.99 \\
\hline \multirow[t]{10}{*}{ wbp } & 10 & 10 & 40 & 7.3 & 0.00 & 7.3 & 0.00 & 7.3 & 0.00 \\
\hline & 10 & 20 & 70 & 8.7 & 0.00 & 8.7 & 0.02 & 8.7 & 0.00 \\
\hline & 10 & 30 & 100 & 9.3 & 0.00 & 9.3 & 0.03 & 9.3 & 0.00 \\
\hline & 15 & 15 & 60 & 11.1 & 0.00 & 11.1 & 0.04 & 11.1 & 0.00 \\
\hline & 15 & 30 & 120 & 13.1 & 0.00 & 13.1 & 0.18 & 13.1 & 0.01 \\
\hline & 20 & 10 & 40 & 15.1 & 0.00 & 15.1 & 0.03 & 15.1 & 0.00 \\
\hline & 20 & 20 & 90 & 15.4 & 0.00 & 15.4 & 0.13 & 15.4 & 0.01 \\
\hline & 30 & 10 & 40 & 23.2 & 0.08 & 23.2 & 0.06 & 23.2 & 0.00 \\
\hline & 30 & 15 & 60 & 23.0 & 0.19 & 23.0 & 0.14 & 23.0 & 0.01 \\
\hline & 30 & 30 & 140 & 24.5 & 0.98 & 24.5 & 0.74 & 24.5 & 1.20 \\
\hline
\end{tabular}

(C) 2014 The Authors International Transactions in Operational Research (C) 2014 International Federation of Operational Research Societies 
Table 7

Computational results: Miller and Wilson benchmarks (individual instances)

\begin{tabular}{|c|c|c|c|c|c|c|c|c|c|c|}
\hline \multirow[b]{2}{*}{ Instance } & \multirow[b]{2}{*}{$I$} & \multirow[b]{2}{*}{$P$} & \multicolumn{2}{|c|}{ BRKGA } & \multicolumn{2}{|l|}{ DP1 } & \multicolumn{2}{|l|}{ DP2 } & \multicolumn{2}{|l|}{ AGA } \\
\hline & & & MOSP & $T$ (seconds) & MOSP & $T$ (seconds) & MOSP & $T$ (seconds) & MOSP & $T$ (seconds) \\
\hline Miller & 20 & 40 & 13 & 0.0 & 13 & 0.6 & - & - & 13 & 2.68 \\
\hline GP1 & 50 & 50 & 45 & 0.0 & 45 & 0.0 & - & - & 45 & 0.02 \\
\hline GP2 & 50 & 50 & 40 & 0.0 & 40 & 0.0 & - & - & 40 & 0.04 \\
\hline GP3 & 50 & 50 & 40 & 0.0 & 40 & 0.0 & - & - & 40 & 0.24 \\
\hline GP4 & 50 & 50 & 30 & 0.0 & 30 & 0.0 & - & - & 30 & 0.02 \\
\hline GP5 & 100 & 100 & 95 & 0.5 & 95 & 0.1 & - & - & 95 & 0.44 \\
\hline GP6 & 100 & 100 & 75 & 0.7 & 75 & 0.1 & - & - & 75 & 0.62 \\
\hline GP7 & 100 & 100 & 75 & 0.7 & 75 & 0.1 & - & - & 75 & 0.58 \\
\hline GP8 & 100 & 100 & 60 & 0.7 & 60 & 0.2 & - & - & 60 & 0.58 \\
\hline NWRS1 & 10 & 20 & 3 & 0.0 & 3 & 0.0 & - & - & 3 & 0 \\
\hline NWRS2 & 10 & 20 & 4 & 0.0 & 4 & 0.0 & - & - & 4 & 0 \\
\hline NWRS3 & 15 & 25 & 7 & 0.0 & 7 & 0.0 & - & - & 7 & 0 \\
\hline NWRS4 & 15 & 25 & 7 & 0.0 & 7 & 0.0 & - & - & 7 & 0 \\
\hline NWRS5 & 20 & 30 & 12 & 0.0 & 12 & 0.0 & - & - & 12 & 0 \\
\hline NWRS6 & 20 & 30 & 12 & 0.0 & 12 & 0.0 & - & - & 12 & 0 \\
\hline NWRS7 & 25 & 60 & 10 & 0.0 & 10 & 0.0 & - & - & 10 & 0 \\
\hline NWRS8 & 25 & 60 & 16 & 0.0 & 16 & 2.1 & - & - & 16 & 6 \\
\hline SP1 & 25 & 25 & 9 & 0.0 & 9 & 0.0 & - & - & 9 & 0.45 \\
\hline SP2 & 50 & 50 & 19 & 0.0 & 19 & 1650.0 & 19 & 0.0 & 19 & 10.9 \\
\hline SP3 & 75 & 75 & 34 & 0.2 & 36 & $\sim 3600$ & 34 & 0.4 & 34 & 36.7 \\
\hline SP4 & 100 & 100 & 53 & 0.6 & 56 & $\sim 14400$ & 53 & 9.1 & 53 & 81.0 \\
\hline
\end{tabular}

For the population size, we obtained good results by indexing it to the dimension of the problem, that is, we used small-sized populations for small problems and larger populations for larger problems. The configuration presented in Table 5 was held constant for all experiments and all problem instances. The computational results presented in the next section demonstrate that this configuration not only provides excellent results in terms of solution quality but is also very robust.

\subsection{Computational results}

Algorithm $B R K G A$ was implemented in $\mathrm{C}++$ and the experiments were carried out on a computer with Intel Core i7-2630QM @2.0 GHZ CPU running the Linux operating system with Fedora release 16. Before running the $B R K G A$, we applied a preprocessing step to each instance as described in Becceneri et al. (2004).

\section{Literature instances}

Due to the nondeterministic nature of BRKGA, 10 runs have been considered for each instance, and the best results are used for comparison. Tables 6-11 present the results obtained by the BRKGA and some of the other approaches for the various instance classes (6141 instances) included in the 
Table 8

Computational results: Faggioli and Bentivoglio's (1998) instances

\begin{tabular}{|c|c|c|c|c|c|c|c|c|}
\hline$I$ & $P$ & & & & & & & \\
\hline$n$ & $m$ & OPT & BRKGA & GHP & $\mathrm{TS}$ & GLS & YUEN3 & YUEN5 \\
\hline 10 & 10 & 5.5 & 5.5 & 5.5 & 513.5 & 5.5 & 5.8 & 5.7 \\
\hline & 15 & 6.6 & 6.6 & 6.6 & 6.6 & 6.6 & 7.0 & 7.0 \\
\hline & 20 & 7.5 & 7.5 & 7.7 & 7.7 & 7.5 & 7.5 & 7.8 \\
\hline & 25 & 8.0 & 8.0 & 8.0 & 8.0 & 8.0 & 8.2 & 8.0 \\
\hline & 30 & 7.8 & 7.8 & 7.8 & 7.8 & 7.8 & 8.2 & 7.9 \\
\hline & 40 & 8.4 & 8.4 & 8.4 & 8.4 & 8.4 & 8.6 & 8.4 \\
\hline 20 & 10 & 6.2 & 6.2 & 6.6 & 6.2 & 6.2 & 6.8 & 6.7 \\
\hline & 15 & 7.2 & 7.2 & 7.5 & 7.2 & 7.5 & 8.4 & 8.3 \\
\hline & 20 & 8.5 & 8.5 & 8.8 & 8.7 & 8.6 & 10.1 & 9.5 \\
\hline & 25 & 9.8 & 9.8 & 9.9 & 9.8 & 9.9 & 11.4 & 10.9 \\
\hline & 30 & 11.1 & 11.1 & 11.4 & 11.2 & 11.2 & 12.7 & 12.1 \\
\hline & 40 & 13,0 & 13.0 & 13.1 & 13.1 & 13.1 & 14.8 & 13.7 \\
\hline 30 & 10 & 6.1 & 6.1 & 6.4 & 6.1 & 6.2 & 7.0 & 7.0 \\
\hline & 15 & 7.4 & 7.3 & 8.0 & 7.4 & 7.6 & 9.1 & 8.6 \\
\hline & 20 & 8.8 & 8.8 & 9.8 & 9.2 & 8.9 & 10.8 & 10.2 \\
\hline & 25 & 10.5 & 10.5 & 11.1 & 10.7 & 10.6 & 12.8 & 12.2 \\
\hline & 30 & 12,2 & 12.2 & 13.0 & 12.6 & 12.2 & 14.7 & 13.6 \\
\hline & 40 & 14,5 & 14.5 & 15.0 & 14.7 & 14.6 & 17.3 & 15.9 \\
\hline 40 & 10 & 7.7 & 7.7 & 7.9 & 7.7 & 7.7 & 8.0 & 7.9 \\
\hline & 15 & 7.3 & 7.2 & 8.2 & 7.3 & 7.4 & 8.4 & 8.1 \\
\hline & 20 & 8.5 & 8.5 & 9.5 & 8.6 & 8.7 & 10.4 & 9.9 \\
\hline & 25 & 10.3 & 10.3 & 11.6 & 10.7 & 10.6 & 13.1 & 11.6 \\
\hline & 30 & 12.1 & 12.1 & 13.4 & 12.6 & 12.4 & 15.1 & 14.6 \\
\hline & 40 & 15.0 & 14.9 & 15.8 & 15.3 & 15.3 & 18.5 & 16.9 \\
\hline 50 & 10 & 8.2 & 8.2 & 8.4 & 8.2 & 8.2 & 8.5 & 8.4 \\
\hline & 15 & 7.4 & 7.4 & 8.4 & 7.6 & 7.6 & 8.4 & 8.1 \\
\hline & 20 & 7.9 & 7.9 & 9.2 & 8.0 & 8.2 & 9.7 & 9.2 \\
\hline & 25 & 10.0 & 10.0 & 11.2 & 10.1 & 10.2 & 12.3 & 12.0 \\
\hline & 30 & 11.2 & 11.2 & 12.4 & 12.0 & 11.8 & 14.9 & 13.5 \\
\hline & 40 & 14.6 & 14.6 & 16.8 & 15.3 & 14.9 & 18.5 & 17.5 \\
\hline
\end{tabular}

set "Literature instances." In the tables, each row is associated with a class of aggregated instances or an individual instance. The first columns define instance name and size, optimal values, etc. The columns denoted by MOSP represent the best MOSP found by the corresponding approach. In terms of computational times, we cannot make any fair and meaningful comment since all the other approaches were implemented with different programming languages and tested on computers with different computing power. Hence, to avoid discussion about the different computer speed used in the tests, we limit ourselves to reporting the average running times per run for BRKGA, while for each of the other algorithms we only report, when available, the reported running times. When available, the columns with header $T$ (seconds) represent the running time of the corresponding approaches, in seconds.

BRKGA dominates all other approaches with respect to the quality of the solution and is very competitive in terms of running time. BRKGA always finds the optimal or best-known solution of 




(C) 2014 The Authors.

International Transactions in Operational Research (c) 2014 International Federation of Operational Research Societies 
Table 10

Computational results: real instances from company "A"

\begin{tabular}{|c|c|c|c|c|c|c|c|}
\hline & \multirow{2}{*}{$\frac{I}{n}$} & \multicolumn{6}{|l|}{$P$} \\
\hline & & $m$ & OPT & BRKGA & $T$ (seconds) & AGA & $T$ (seconds) \\
\hline A_AP-9.d_10 & 20 & 13 & 6 & 6 & 0.05 & 6 & 0.12 \\
\hline A_AP-9.d_3 & 20 & 16 & 6 & 6 & 0.05 & 6 & 0.17 \\
\hline A_FA+AA-_15 & 68 & 18 & 9 & 9 & 0.23 & 9 & 0.63 \\
\hline A_FA+AA-_2 & 75 & 19 & 11 & 11 & 0.15 & 11 & 0.68 \\
\hline A_AP-9.d_6 & 31 & 20 & 5 & 5 & 0.15 & 5 & 0.40 \\
\hline A_FA+AA-_12 & 75 & 20 & 9 & 9 & 0.23 & 9 & 0.77 \\
\hline A_AP-9.d_11 & 27 & 21 & 6 & 6 & 0.15 & 6 & 0.42 \\
\hline A_FA+AA-_6 & 79 & 21 & 13 & 13 & 0.28 & 13 & 1.20 \\
\hline A_FA+AA-_8 & 82 & 28 & 11 & 11 & 0.60 & 12 & 2.65 \\
\hline A_FA+AA-_11 & 99 & 28 & 11 & 11 & 0.65 & 11 & 2.92 \\
\hline A_FA+AA-_1 & 107 & 37 & 12 & 12 & 1.38 & 12 & 7.58 \\
\hline A_FA+AA-_13 & 134 & 37 & - & 17 & 1.45 & 17 & 11.82 \\
\hline
\end{tabular}

Table 11

Computational results: real instances from company "B"

\begin{tabular}{llllllll}
\hline & $\frac{I}{n}$ & $\frac{P}{m}$ & OPT & BRKGA & $T$ (seconds) & AGA & $T$ (seconds) \\
\hline B_39Q18_82 & 14 & 10 & 5 & 5 & 0.00 & 5 & 0.00 \\
B_42F22_93 & 18 & 10 & 5 & 5 & 0.05 & 5 & 0.04 \\
B_22X18_50 & 14 & 11 & 10 & 10 & 0.03 & 10 & 0.05 \\
B_18AB1_32 & 15 & 11 & 6 & 6 & 0.03 & 6 & 0.05 \\
B_CARLET_137 & 14 & 12 & 5 & 5 & 0.03 & 5 & 0.00 \\
B_12F18_11 & 21 & 15 & 6 & 6 & 0.08 & 6 & 0.16 \\
B_18CR1_33 & 20 & 18 & 4 & 4 & 0.03 & 4 & 0.17 \\
B_GTM18A_139 & 24 & 20 & 5 & 5 & 0.10 & 5 & 0.30 \\
B_23B25_52 & 29 & 21 & 5 & 5 & 0.10 & 5 & 0.39 \\
B_12M18_12 & 31 & 22 & 6 & 6 & 0.15 & 6 & 0.00 \\
B_CUC28A_138 & 37 & 26 & 6 & 6 & 0.10 & 6 & 0.00 \\
B_REVAL_145 & 60 & 49 & 7 & 7 & 1.28 & 7 & 9.37 \\
\hline
\end{tabular}

MOSP in reasonable running time (often negligible), while the computational effort as well as the quality of the solutions of DP1 degrade with large-sized instances SP3 and SP4. DP2 overcomes this issue and solves instances SP2, SP3, and SP4 very quickly. For most of the instances, the BRKGA obtains the best solution before the 10th generation.

\section{Harder instances}

In this section, we use a total of 150 harder instances to evaluate the performance of BRKGA. These instances were generated by De Giovanni et al. (2013) using the same random generator as Chu and Stuckey (2009). For each triplet defined by the number of patterns chosen in the set $\{50,100,150\}$, 


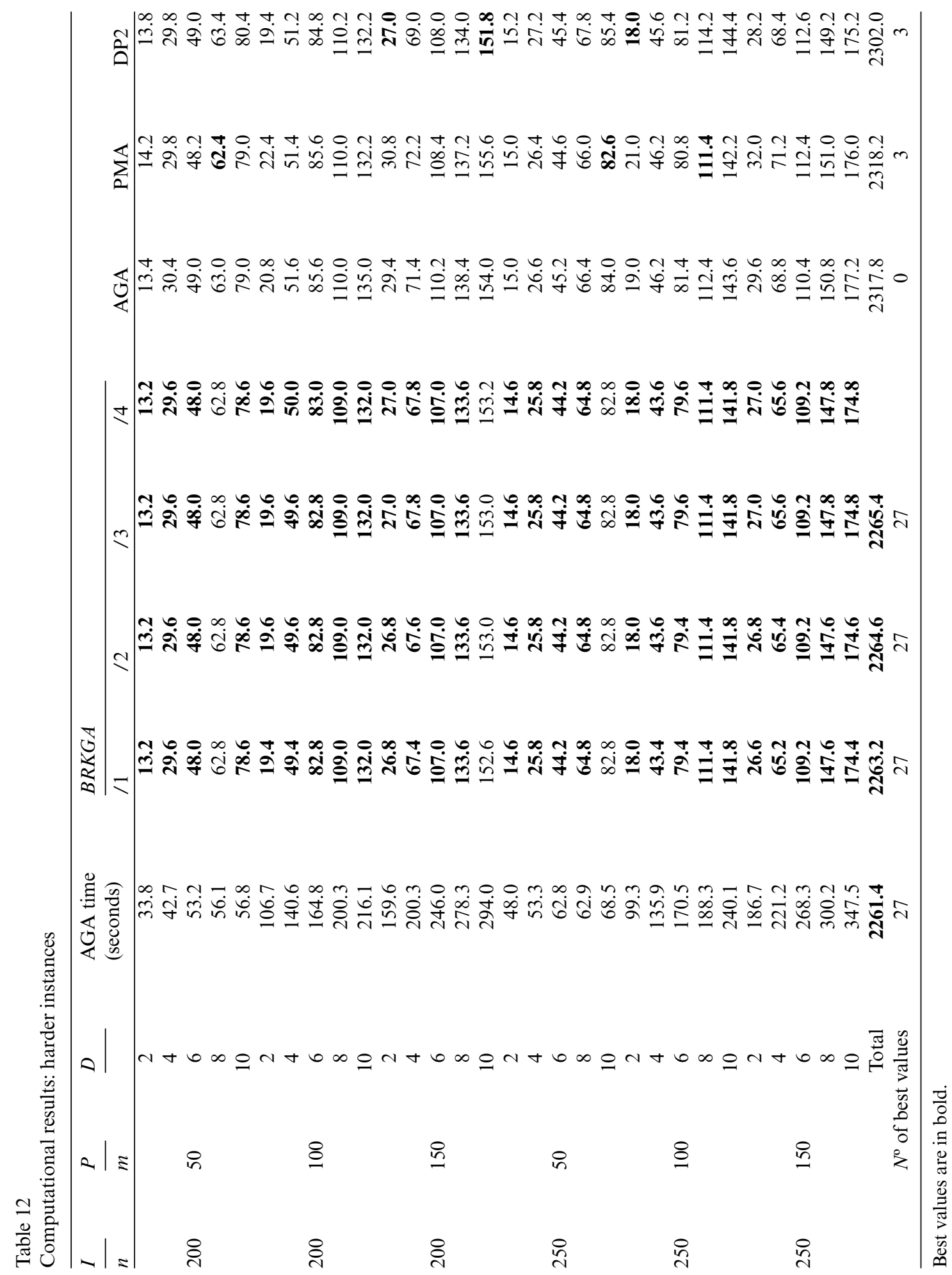

(C) 2014 The Authors.

International Transactions in Operational Research (c) 2014 International Federation of Operational Research Societies 
Table 13

Description of additional experiments

\begin{tabular}{ll}
\hline Experiment & Description \\
\hline GA & Run plain BRKGA with chromosome adjustment \\
GA-M & Run plain BRKGA with chromosome adjustment and with the modified MOSP \\
GA-LS & Run plain BRKGA with chromosome adjustment and with the local search \\
GA-LS-M & Run plain BRKGA with chromosome adjustment and the local search and modified MOSP \\
& (i.e., BRKGA) \\
\hline
\end{tabular}

the number of item types chosen in the set $\{200,250\}$, and the density (average number of item types per pattern) chosen in the set $\{2,4,6,8,10\}$, five instances were generated. As a postcondition, any instance where the customer graph can be divided into separate components is discarded to avoid relatively trivial instances. We compare BRKGA against AGA, DP2, and PMA using the results published in De Giovanni et al. (2013). DP2 and PMA were run on the same configuration as AGA by De Giovanni et al. (2013). In order to make the comparison as fair as possible and to take into account the nondeterministic nature of AGA and PMA (and the potentially long computational time of DP2 as an exact approach), De Giovanni et al. (2013) tested AGA five times for each instance and recorded the average computational time; then, DP2 and PMA were run and stopped after the same computational time, storing the best (if not provably optimal) solution found so far (PMA was repeated five times per instance).

Summary results are given in Table 12. Each row shows the results for each group of five instances with the same number of item types $(I)$, number of patterns $(P)$, and density $(D)$. The columns corresponding to AGA and PMA report the minimum value of MOSP over the five runs. The column corresponding to DP2 reports the minimum value of MOSP obtained within the time limit. For the BRKGA, we include four columns $/ 1, / 2, / 3$, and $/ 4$ that correspond to the minimum value of MOSP obtained by BRKGA, over five runs, when we use as computational time the time limit used by AGA was divided by 1,2, 3, and 4, respectively.

Table 12 shows that $B R K G A$ consistently produces solutions that outperform the algorithms $A G A, P M A$, and $D P 2$ in 27 of all the 30 subgroups, even when a fourth of the computational time limit is used. A statistical analysis of the results using the Wilcoxon test for the matched pairs $B R K G A<A G A, B R K G A<P M A$, and $B R K G A<D P 2$ shows that $B R K G A$ is significantly better than all the other algorithms obtaining $p$-values for all paired comparisons smaller than 0.001 .

\section{Becceneri instances}

In this subsection, we compare BRKGA against the low-order polynomial time $A S$ heuristic for MOSP proposed by Ashikaga and Soma (2009). The comparison uses the Becceneri set of instances generated by Becceneri (1999), which covers the most common practical industrial scenario applications. The tests follow the notation used by Becceneri et al. (2004), that is, $n \times m$ that stands, respectively, for the number of items and number of patterns, and $C$ is the maximum 
으

年

$\stackrel{+}{+}$

$m$

으

กิ

กิ


in

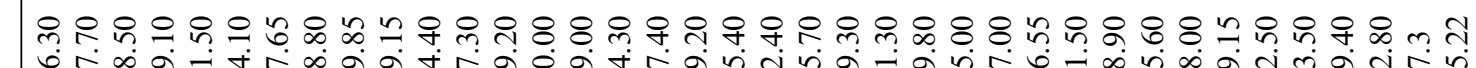
으





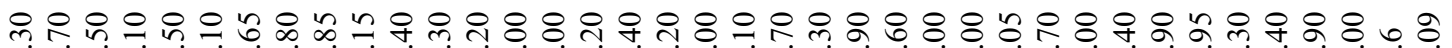
৩


ن

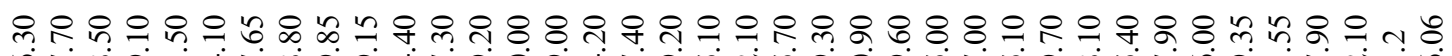
نं



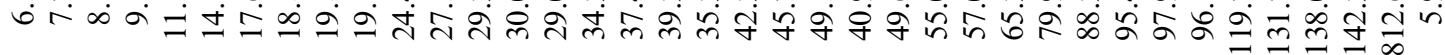

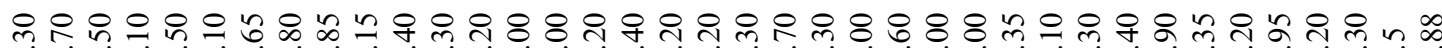

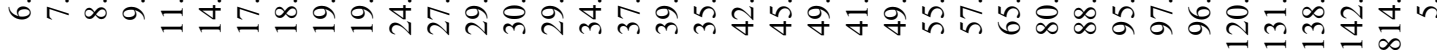

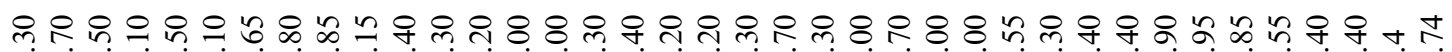

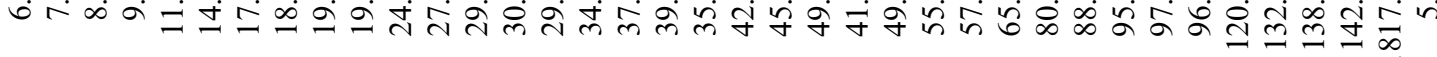



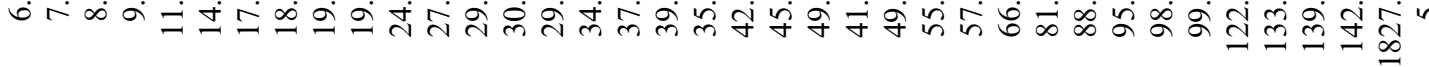

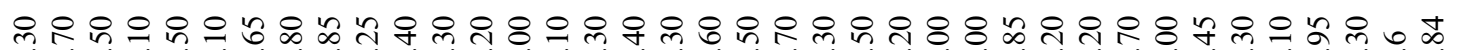
or in

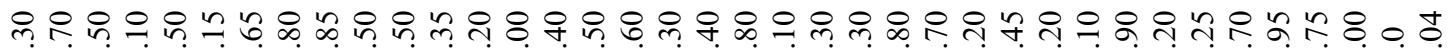
ن


$\&$



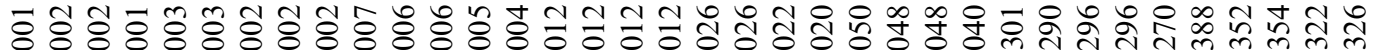

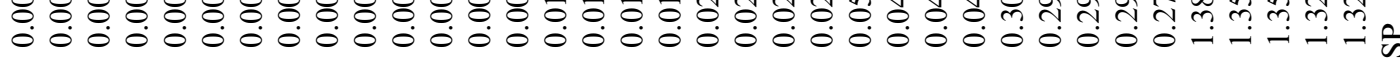

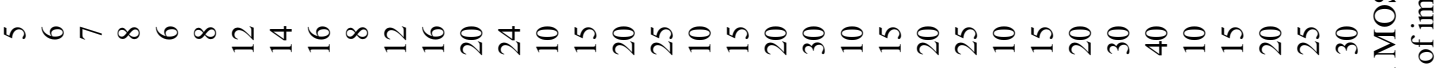

으

ㄱำ

ㄱ

군

8

으

은

으

ㅇำ

r

요

오

8

은

퓽

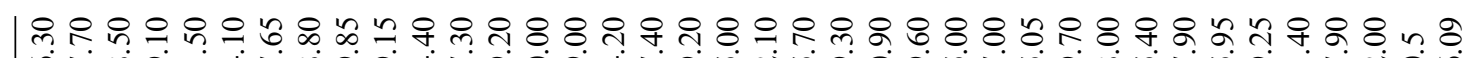




Table 15

Computational results: BRKGA component performance

\begin{tabular}{|c|c|c|c|c|c|c|}
\hline \multirow{2}{*}{$\frac{I}{n}$} & \multirow{2}{*}{$\frac{P}{m}$} & \multirow[b]{2}{*}{$D$} & \multirow[b]{2}{*}{ GA } & \multirow[b]{2}{*}{ GA-M } & \multirow[b]{2}{*}{ GA-LS } & \multirow{2}{*}{$\begin{array}{l}\text { (BRKGA) } \\
\text { GA-LS-M }\end{array}$} \\
\hline & & & & & & \\
\hline & & 2 & 19.4 & 15.2 & 13.0 & 13.2 \\
\hline & & 4 & 35.8 & 31.6 & 29.8 & 29.8 \\
\hline \multirow[t]{5}{*}{200} & 50 & 6 & 52.6 & 49.2 & 48.4 & 48.0 \\
\hline & & 8 & 68.0 & 64.4 & 62.8 & 62.8 \\
\hline & & 10 & 83.2 & 80.2 & 79.0 & 78.6 \\
\hline & & 2 & 33.2 & 25.2 & 19.8 & 19.6 \\
\hline & & 4 & 60.8 & 53.8 & 50.6 & 50.0 \\
\hline \multirow[t]{5}{*}{200} & 100 & 6 & 94.6 & 88.2 & 84.0 & 82.8 \\
\hline & & 8 & 119.0 & 113.0 & 109.8 & 109.0 \\
\hline & & 10 & 141.8 & 134.6 & 133.4 & 132.0 \\
\hline & & 2 & 47.8 & 36.2 & 28.2 & 26.8 \\
\hline & & 4 & 85.6 & 75.6 & 69.0 & 67.4 \\
\hline \multirow[t]{5}{*}{200} & 150 & 6 & 122.8 & 113.0 & 108.2 & 107.0 \\
\hline & & 8 & 149.0 & 141.2 & 134.8 & 133.6 \\
\hline & & 10 & 163.0 & 159.4 & 154.8 & 152.6 \\
\hline & & 2 & 20.8 & 17.0 & 14.6 & 14.6 \\
\hline & & 4 & 32.2 & 27.8 & 26.0 & 25.8 \\
\hline \multirow[t]{5}{*}{250} & 50 & 6 & 50.0 & 45.8 & 44.2 & 44.2 \\
\hline & & 8 & 70.8 & 66.6 & 65.0 & 64.8 \\
\hline & & 10 & 89.0 & 84.6 & 82.8 & 82.8 \\
\hline & & 2 & 33.8 & 24.0 & 18.4 & 18.0 \\
\hline & & 4 & 58.6 & 48.8 & 44.6 & 43.6 \\
\hline \multirow[t]{5}{*}{250} & 100 & 6 & 90.8 & 84.4 & 80.8 & 79.4 \\
\hline & & 8 & 121.0 & 116.2 & 112.8 & 111.4 \\
\hline & & 10 & 150.2 & 144.8 & 143.0 & 141.8 \\
\hline & & 2 & 48.2 & 37.8 & 28.6 & 26.6 \\
\hline & & 4 & 83.2 & 75.0 & 66.8 & 65.2 \\
\hline \multirow[t]{3}{*}{250} & 150 & 6 & 125.8 & 118.0 & 111.4 & 109.2 \\
\hline & & 8 & 163.2 & 154.6 & 150.4 & 147.6 \\
\hline & & 10 & 189.4 & 180.4 & 176.8 & 174.6 \\
\hline Total & & & 2603.6 & 2406.6 & 2291.8 & 2262.8 \\
\hline \multicolumn{3}{|c|}{ Percentage of improvement over GA } & & 7.57 & 11.98 & 13.09 \\
\hline
\end{tabular}

number of items allocated to a pattern. For each group, a sample of 20 random instances was generated.

In the test, we let BRKGA run once for 50 generations and registered the value of MOSP at the end of the first generation and then every five generations. Summary results are given in Table 14, where each row shows the results for the average MOSP values over the instances in the group. The column "Time" corresponds to the time used by BRKGA for one generation while the column $A S$ represents the average MOSP obtained by the AS heuristic. The columns with headings 1, 5, ..,50 correspond to the values of MOSP obtained by the BRKGA after the number of generations in the corresponding heading.

After only one generation the BRKGA obtains, for all the 36 groups, MOSP values that are equal to or better than the values obtained by the AS heuristic. The MOSP values obtained by BRKGA

(C) 2014 The Authors International Transactions in Operational Research (C) 2014 International Federation of Operational Research Societies 
over all the groups on the first generation correspond already to an improvement of $4.04 \%$ over the values of the AS heuristic, and as the number of generations increase the improvement goes up to $6.09 \%$. BRKGA always finds better values than AS in reasonable running time (often negligible). As the size of the instance increases, the computational time increases quite slowly.

\section{Impact of each component on the BRKGA performance}

To investigate the impact of each of the components included in BRKGA (GA, local search, and modified MOSP) on its performance, we conducted the additional experiments described in Table 13 .

Each experiment was run five times for 100 generations on the 150 harder instances presented in Section "Harder instances." Summary results are given in Table 15. Each row shows the results for each group of five instances with the same number of item types $(I)$, number of patterns $(P)$, and density $(D)$. The columns corresponding to GA, GA-M, GA-LS, and GA-LS-M report the minimum value of MOSP over the five runs.

From Table 15, it is clear that the plain BRKGA (GA) does not perform well since it produces an overall MOSP increase of $13 \%$ with respect to the full algorithm (BRKGA). The combinations of the plain BRKGA (GA) with the modified MOSP (GA-M) and with the local search (GA-LS) produce better results. Nevertheless, they are $5.5 \%$ and $1.1 \%$, respectively, above the ones produced by the full BRKGA (GA-LS-M). Combining the plain BRKGA with both the local search and modified MOSP into GA-LS-M results in the best values of MOSP.

\section{Concluding remarks}

In this paper, we addressed the MOSP that consists of determining a sequence of cutting patterns that minimize the maximum number of open stacks during the cutting process. The approach proposed combines a $B R K G A$ and local search procedure for generating the sequence of cutting patterns. A new fitness function for evaluating the quality of the solutions is also proposed. Computational tests are presented using 6141 available instances taken from the literature. The high quality of the solutions obtained validate the proposed approach.

The new approach is extensively tested on more than 7000 problem instances and compared with other approaches published in the literature. The computational experiments results demonstrate that the new approach consistently equals or outperforms the other approaches.

\section{Acknowledgments}

This work has been partially supported by projects PTDC/EGE-GES/117692/2010 and NORTE07-0124-FEDER-000057 funded by the North Portugal Regional Operational Programme (ON.2O Novo Norte), under the National Strategic Reference Framework (NSRF), through the European Regional Development Fund (ERDF) and the Programme COMPETE; and by national funds, through the Portuguese funding agency, Fundação para a Ciência e a Tecnologia (FCT). We would like to thank Luigi De Giovanni, Fernando Masanori Ashikaga, and Geoffrey Chu for sending us instances for the problem. 


\section{References}

Ashikaga, F.M., Soma, N.Y., 2009. A heuristic for the minimization of open stacks problem. Pesquisa Operacional 29, 439-450.

Banda, M.G., Stuckey, P.J., 2007. Dynamic programming to minimize the maximum number of open stacks. INFORMS Journal on Computing 19, 607-617.

Bean, J.C., 1994. Genetics and random keys for sequencing and optimization. ORSA Journal on Computing 6, 154-160.

Becceneri, J., 1999. O problema de sequenciamento de padr oes para minimizaçao do número máximo de pilhas abertas em ambientes de corte industriais. Ph.D. thesis, Engenharia Eletrônica e Computação, ITA/CTA, São José dos Campos.

Becceneri, J.C., Yanasse, H.H., Soma, N.Y., 2004. A method for solving the minimization of the maximum number of open stacks problem within a cutting process. Computers \& Operations Research 31, 2315-2332.

Chu, G., Stuckey, P.J., 2009. Minimizing the maximum number of open stacks by customer search. In Gent, I.P. (ed.) Principles and Practice of Constraint Programming - CP 2009, Vol. 5732 of Lecture Notes in Computer Science, Springer, Berlin Heidelberg, pp. 242-257.

De Giovanni, L., Massi, G., Pezzella, F., 2010. Preliminary computational experiments with a genetic algorithm for the open stacks problem. Technical Report, Dipartimento di Matematica Pura ed Applicata Università degli studi di Padova, Padova, Italy.

De Giovanni, L., Massi, G., Pezzella, F., 2013. An adaptive genetic algorithm for large-size open stack problems. International Journal of Production Research 51, 682-697.

Faggioli, E., Bentivoglio, C.A., 1998. Heuristic and exact methods for the cutting sequencing problem. European Journal of Operational Research 110, 564-575.

Fink, A., Voß, S., 1999. Applications of modern heuristic search methods to pattern sequencing problems. Computers and Operations Research 26, 17-34.

Gonçalves, J.F., Almeida, J.R., 2002. A hybrid genetic algorithm for assembly line balancing. Journal of Heuristics 8 , $629-642$.

Gonçalves, J.F., Mendes, J.J.M., Resende, M.G.C., 2005. A hybrid genetic algorithm for the job shop scheduling problem. European Journal of Operational Research 167, 77-95.

Gonçalves, J.F., Mendes, J.J.M., Resende, M.G.C., 2009. A genetic algorithm for the resource constrained multi-project scheduling problem. European Journal of Operational Research 189, 1171-1190.

Gonçalves, J.F., Resende, M.G.C., 2004. An evolutionary algorithm for manufacturing cell formation. Computers and Industrial Engineering 47, 247-273.

Gonçalves, J.F., Resende, M.G.C., 2011. Biased random-key genetic algorithms for combinatorial optimization. Journal of Heuristics 17, 487-525.

Gonçalves, J.F., Resende, M.G.C., 2012. A parallel multi-population biased random-key genetic algorithm for a container loading problem. Computers \& Operations Research 39, 179-190.

Gonçalves, J.F., Resende, M.G.C., 2013. A biased random key genetic algorithm for 2D and 3D bin packing problems. International Journal of Production Economics 145, 500-510.

Gonçalves, J.F., Resende, M.G.C., 2014. An extended Akers graphical with a biased random-key genetic algorithm for job-shop scheduling. International Transactions in Operational Research 21, 215-246.

Gonçalves, J.F., Resende, M.G.C., Mendes, J.J.M., 2011. A biased random-key genetic algorithm with forward-backward improvement for the resource constrained project scheduling problem. Journal of Heuristics 17, 467-486.

Gonçalves, J.F., Sousa, P.S.A., 2011. A genetic algorithm for lot sizing and scheduling under capacity constraints and allowing backorders. International Journal of Production Research 49, 2683-2703.

Hu, Y.H., Chen, S.J., 1990. Gm plan: a gate matrix layout algorithm based on artificial intelligence planning techniques. IEEE Transactions on Computer-Aided Design of Integrated Circuits and Systems 9, 836-845.

Limeira, M., 1998. Desenvolvimento de um algoritmo exato para a solução de um problema de sequenciamento de padrões de corte. 1998. Ph.D. thesis, Dissertação (Mestrado em Computação Aplicada), Instituto Nacional de Pesquisas Espaciais, São José dos Campos.

Linhares, A., Yanasse, H.H., 2002. Connections between cutting-pattern sequencing, VLSI design, and flexible machines. Computers \& Operations Research 29, 1759-1772.

(C) 2014 The Authors

International Transactions in Operational Research (C) 2014 International Federation of Operational Research Societies 
Linhares, A., Yanasse, H.H., Torreao, J.R., 1999. Linear gate assignment: a fast statistical mechanics approach. IEEE Transactions on Computer-Aided Design of Integrated Circuits and Systems 18, 1750-1758.

Lins, S., 1989. Traversing trees and scheduling tasks for duplex corrugator machines. Pesquisa Operacional 9, 40-54.

Mendes, A., Linhares, A., 2004. A multiple-population evolutionary approach to gate matrix layout. International Journal of Systems Science 35, 13-23.

Möhring, R.H., 1990. Graph problems related to gate matrix layout and PLA folding. In Tinhofer, G., Mayr, E., Noltemeier, H., Syslo, M.M. (eds) Computational Graph Theory. Springer, Vienna, pp. 17-51.

Morán-Mirabal, L.F., González-Velarde, J.L., Resende, M.G.C., 2014. Randomized heuristics for the family traveling salesperson problem. International Transactions in Operational Research 21, 41-57.

Oliveira, A.C.M., Lorena, L.A.N., 2002. A constructive genetic algorithm for gate matrix layout problems. IEEE Transactions on Computer-Aided Design of Integrated Circuits and Systems 21, 969-974.

Oliveira, A.C.M., Lorena, L.A.N., 2006. Pattern sequencing problems by clustering search. In Sichman, J.S., Coelho, H., Rezende, S.O. (eds) Advances in Artificial Intelligence - IBERAMIA-SBIA 2006, Vol. 4140 of Lecture Notes in Computer Science. Springer, Berlin, Heidelberg, pp. 218-227.

Smith, B., Gent, I., 2005. Constraint modelling challenge 2005. IJCAI 2005 Fifth Workshop on Modelling and Solving Problems with Constraints, Edinburgh, pp. 1-8.

Spears, W.M., Dejong, K.A., 1991. On the virtues of parameterized uniform crossover. Proceedings of the Fourth International Conference on Genetic Algorithms, San Diego, CA, pp. 230-236.

Yanasse, H., 1996. Minimization of open orders-polynomial algorithms for some special cases. Pesquisa Operacional 16, $1-26$.

Yanasse, H., 1997a. A transformation for solving a pattern sequencing problem in the wood cut industry. Pesquisa Operacional 17, 57-70.

Yanasse, H.H., 1997b. On a pattern sequencing problem to minimize the maximum number of open stacks. European Journal of Operational Research 100, 454-463.

Yanasse, H., Becceneri, J., Soma, N., 1999. Bounds for a problem of sequencing patterns. Pesquisa Operacional 19, 249-277.

Yanasse, H.H., Becceneri, J.C., Soma, N.Y., 2007. Um algoritmo exato com ordenamento parcial para solução de um problema de programação da produção: experimentos computacionais. Gestão \& Produção 14, 353-361.

Yanasse, H., Limeira, M., 2004. Refinements on an enumeration scheme for solving a pattern sequencing problem. International Transactions in Operational Research 11, 277-292.

Yanasse, H.H., Senne, E.L.F., 2010. The minimization of open stacks problem: a review of some properties and their use in pre-processing operations. European Journal of Operational Research 203, 559-567.

Yuen, B.J., 1991. Heuristics for sequencing cutting patterns. European Journal of Operational Research 55, 183-190.

Yuen, B.J., 1995. Improved heuristics for sequencing cutting patterns. European Journal of Operational Research 87, 57-64.

Yuen, B.J., Richardson, K.V., 1995. Establishing the optimality of sequencing heuristics for cutting stock problems. European Journal of Operational Research 84, 590-598.

(c) 2014 The Authors.

International Transactions in Operational Research @ 2014 International Federation of Operational Research Societies 